\title{
Announcements of the Board of ORCA
}

Statement of ORCA on Measures for Preventing Dental Caries

The General Assembly on 17th July 1970 in Debrecen adopted the following two statements translated into German and French by an ad hoc editorial committee nominated by the Board A primary aim of the European Organisation for Caries Research (ORCA) is to promote the application of scientifically based and proven methods for preventing dental decay. This statement outlines the most effective measures of achieving this end according to present knowledge.

It is important to improve dietary habits and oral hygiene. High and frequent intakes of sugar and sweets, especially between meals, should be discouraged as the frequent consumption of sugared confections is the main factor in the production of substances harmful to teeth by the bacteria on the tooth surfaces. Good oral hygiene is beneficial both to gums and teeth but demands more continuing effort than most individuals are willing to exert.

In the light of the vast amount of well documented scientific evidence ORCA supports unequivocally fluoridation of water supplies as the most practicable and most effective public measure for inhibiting tooth decay. Since water fluoridation does not completely prevent this disease and is not universally applicable, research into other measures of prevention must be continued. Encouraging results have been obtained with other vehicles for fluoride such as tablets or domestic salt.

Part of the protective action of fluoride is exerted at the tooth surface. Dentifrices, mouth rinses or other topical agents containing fluoride may be used to promote this action and may complement the effects of one of the methods of internal administration.

Statement of ORCA on Fluoridation of Drinking Water

The European Organisation for Caries Research (ORCA) has concluded in the light of evidence from well conducted studies throughout the world that drinking water containing the optimum concentration of fluoride (about one part in a million in most countries) is the most practicable and most effective method of inhibiting tooth decay. If such water is drunk continuously, the effect lasts throughout life and is the same whether the fluoride is present naturally or is added to the water.

2

Statement of ORCA

Many national and international reports prepared by scientific committees have concluded that fluoridation of water is safe. No subsequent data have reduced the validity of this judgment. Well recognised but very rare difficulties, such as those arising from industries handling fluorine containing compounds, are separate problems which can and should be dealt with by the appropriate methods. They therefore do not raise valid objections against bringing the fluoride concentration of the water supply to the optimum level for the control of tooth decay. 